\title{
Numerical study on the shock/combustion interaction of oblique detonation waves
}

\author{
Li Yang ${ }^{\mathrm{a}, \mathrm{b}}$, Lianjie Yue ${ }^{\mathrm{b}, \mathrm{c}, *}$, Qifan Zhang ${ }^{\mathrm{b}, \mathrm{c}}$, Xinyu Zhang ${ }^{\mathrm{b}, \mathrm{c}}$ \\ a School of Aerospace Engineering, Beijing Institute of Technology, Beijing, 100081, China \\ b State Key Laboratory of High Temperature Gas Dynamics, Institute of Mechanics, Chinese Academy of Sciences, Beijing, 100190, China \\ c School of Engineering Sciences, University of Chinese Academy of Sciences, Beijing, 100049, China
}

\section{A R T I C L E I N F O}

Article history:

Received 31 December 2019

Received in revised form 8 February 2020

Accepted 1 April 2020

Available online 12 June 2020

Communicated by Kai Liu

\section{Keywords:}

Oblique detonation wave

Shock/combustion interaction

Shock polar analysis

High-resolution simulation

\begin{abstract}
A B S T R A C T
To determine the shock/combustion interaction structure of a wedge-induced oblique detonation wave, high-order numerical simulations solving the two-dimensional reactive Euler equations embedded with a two-step chemical kinetic model have been performed. In this study, various dimensionless heat release amounts ranging from 40.0 to 52.5 are selected to investigate the flow configuration. The computational results show that four types of typical shock/combustion interactions, namely, Type VI, I, V and IV (following the classification of shock/shock interaction proposed by Edney), can be observed. The detailed structures and characteristics of the interaction types are presented and shown to be different than the classic shock/shock interaction. Additional insight into the transition principles of different shock/combustion interactions is illustrated through shock polar analysis.
\end{abstract}

(c) 2020 Elsevier Masson SAS. All rights reserved.

\section{Introduction}

Advanced propulsion devices should be explored due to the rigorous requirements of hypersonic aircraft, which can be used in commercial aviation, space exploration and defense technology [1]. Compared with traditional propulsion systems, such as rockets and turbo engines, researchers have proposed and developed novel concepts in chemical propulsion fields [2-9], e.g., scramjets, pulsed detonation engines, oblique detonation wave engines (ODWEs), rotating detonation engines and combined-cycle propulsion engines, which are considered the future of airbreathing hypersonic propulsion technology. With a long period of development, currently, scramjets can nearly meet the demands of actual engineering applications, and the combustion characteristics in combustors, such as mode transition, combustion unsteadiness, flame-holder and so on, have been studied widely [10-14]. Theoretically, the advantage of detonative combustion over deflagrative combustion would significantly elevate the propulsion performance, although the feasibility of detonation propulsion has been indirectly demonstrated;

\footnotetext{
* Corresponding author at: State Key Laboratory of High Temperature Gas Dynamics, Institute of Mechanics, Chinese Academy of Sciences, Beijing, 100190, China. E-mail address: yuelj@imech.ac.cn (L. Yue).
}

it is well known that detonative combustion exhibits an important benefit, i.e., the liberation of chemical energy is complete, prompt and confined to a very narrow region. The combustor length of ODWEs is approximately one-fifth that of scramjets, and thus ODWEs require less excess fuel for cooling [15]. As a potential alternative, ODWE research has received vast amounts of attention and remains an active field.

Two types of oblique detonation wave (ODW) evolution phenomena have been confirmed [16-20]: 1) the direct initiation of the detonation wave within the stagnation region at the beginning of the boundary layer, called prompt ODW; and 2) delayed ODW, which is an oblique shock wave (OSW)-to-ODW transition that occurs at a distance from the leading edge of the wedge (this distance is orders of magnitude greater than the boundary layer thickness). The flow configuration structure of a delayed ODW typically consists of an inert OSW, a combustion wave (CW), and an oblique detonation front that are united at a triple point, as shown in Fig. 1. Furthermore, a parametric numerical study was conducted, demonstrating the two types of transition structures of delayed ODWs [21], i.e., abrupt transitions and smooth transitions. The ODW front around hypersonic projectiles is divided into four parts [22]: 1) a strong overdriven detonation wave, 2) a weak overdriven detonation wave, 3) a quasi-CJ detonation wave, and 4) a CJ detonation wave; similar ODW categories also exist in a double wedge-induced ODW, and a quasi-CJ ODW can be established by adjusting the first wedge angle $[23,24]$. 


\section{Nomenclature}

$\begin{array}{ll}\rho & \text { density } \\ u & \text { velocity in the x direction } \\ v & \text { velocity in the y direction } \\ E & \text { total energy per mass } \\ E_{a} & \text { activation energy } \\ p & \text { pressure } \\ T & \text { temperature } \\ Q & \text { dimensionless heat release of chemical reaction } \\ \lambda & \text { chemical reaction progress variable } \\ \omega & \text { chemical reaction rate } \\ K & \text { pre-exponential factor } \\ l_{r e f} & \text { induction zone length } \\ M a & \text { Mach number } \\ R & \text { specific gas constant } \\ \gamma & \text { specific heat ratio } \\ \theta & \text { deflection angle } \\ \beta & \text { wave angle } \\ \Phi & \text { pressure ratio }\end{array}$

\section{Subscripts}

$\infty \quad$ quantities of the free inflow stream
I induction zone

$R \quad$ exothermic reaction zone

1 physical parameters before detonation front

2 physical parameters after detonation front

u unburned state

s post-shock state

b burned state

\section{Abbreviation}

$\begin{array}{ll}\text { OSW } & \text { Oblique shock wave } \\ \text { ODW } & \text { Oblique detonation wave } \\ \text { SL } & \text { Slip line } \\ \text { OODW } & \text { Overdriven oblique detonation wave } \\ \text { CW } & \text { Combustion wave } \\ \text { TW } & \text { Transverse wave } \\ \text { UTP } & \text { Upper triple point } \\ \text { MTP } & \text { Middle triple point } \\ \text { LTP } & \text { Lower triple point } \\ \text { MMS } & \text { Middle Mach stem } \\ \text { LMS } & \text { Lower Mach stem }\end{array}$

Numerous studies have focused on the initiation, sustainability and transition of an ODW. A critical threshold in the initial pressure is required for the establishment of an ODW when a hypervelocity projectile is launched into combustible mixtures; the Damkohler number and the energetic and kinetic limits are introduced to explain the existence of pressure thresholds due to the competition between reactions and flow-quenching effects $[25,26]$. The blunt forebody combined with a wedge plays a positive role in the initiation of an ODW with a wide range of inflow Mach numbers from 8 to 10 ; two means of initiation, wedge-induced initiation and blunt forebody-induced initiation, are determined numerically, and a theoretical analysis based on the critical initiation energy can predict different means of initiation well [27]. The incoming flow at various altitudes and the length of the wedge have an effect on the initiation of a finite wedge-induced ODW, and the initiation of the ODW can be obtained when the characteristic length of oblique wedge is larger than the characteristic length of induction zone [28]. The coupling of the shock wave front and the reaction zone has been investigated computationally. Fully coupled and partially coupled prompt ODWs have been observed, and local explosions occurring in the downstream region of a partially coupled prompt ODW could alter the flow field to the fully coupled prompt ODW back and forth [29]. The dominant factors that influence the decoupled and recoupled characteristics of the ODW are the pre-exponential factor and the activation energy, which are temperature-sensitive parameters in the chemical reaction [30]. In addition, transition criteria for the smooth and abrupt transitions of a delayed ODW have been proposed [21,31-33]. Several physical parameters, e.g., the angle difference between the ODW and the OSW, the critical Mach number, the ratio of the induction time to the total reaction time, the ratio of the inflow velocity to $\mathrm{CJ}$ detonation velocity, the post-OSW-to-post-ODW pressure ratio and the minimum Mach number behind the detonation wave, are adopted to determine the transition patterns.

Studies on the shock/combustion interactions in the transition region of an ODW have not attracted deserved attention. Some numerical computations [34,35] have determined three types of flow field structures, i.e., $\lambda$-shaped, X-shaped and Y-shaped shock configurations, with different inflow Mach numbers. The trajectory of the triple point formed by the intersection of the OSW, ODW, and transverse detonation wave resembles the cellular struc- ture, as observed experimentally [36,37]. Numerical studies have reported that inhomogeneous reactive mixtures have an effect on the flow structures in the transition region of an ODW [38,39], and these structures are controlled by the reactivity gradient, Mach number gradient, post-shock flow field and viscosity. Above all, certain typical structures in the transition region have been acquired; however, a detailed description, categorization and analysis of shock/combustion interactions have not yet been achieved, and the transitions between the above structures have yet to be understood comprehensively.

In this study, a high-order numerical simulation solving the 2D reactive Euler equations equipped with a two-step chemical kinetic model was conducted to investigate the shock/combustion interactions of a wedge-induced ODW. The heat release, $Q$, is the bifurcation variable adopted to examine different flow configurations. Based on the classification of shock/shock interaction proposed by Edney [40], a discussion of the formation and transition of typical interaction structures is provided with a shock/detonation polar curve analysis.

\section{Numerical method and computational setup}

\subsection{Governing equations and numerical schemes}

The two-dimensional reactive Euler equation is adopted in this study with generalized coordinates, that are coupled with a twostep chain-branching chemical kinetics model. The nondimensional governing equations in curvilinear coordinates are summarized as follows [41]:

$J^{-1} \frac{\partial W}{\partial t}+J^{-1} \frac{\partial\left(\xi_{x} F+\xi_{y} G\right)}{\partial \xi}+J^{-1} \frac{\partial\left(\eta_{x} F+\eta_{y} G\right)}{\partial \eta}=J^{-1} S$,

where $\mathrm{W}$ is the conservative solution vector, $\mathrm{F}$ and $\mathrm{G}$ are the convective terms, and $\mathrm{S}$ is the reaction source term as follows:

$$
\begin{aligned}
& \mathrm{W}=\left[\begin{array}{llllll}
\rho & \rho u & \rho v & \rho E & \rho \lambda_{I} & \rho \lambda_{R}
\end{array}\right]^{T}, \\
& F=\left[\begin{array}{llllll}
\rho u & \rho u^{2}+p & \rho u v & (\rho E+p) u & \rho u \lambda_{I} & \rho u \lambda_{R}
\end{array}\right]^{T}, \\
& G=\left[\begin{array}{llllll}
\rho v & \rho u v & \rho v^{2}+p & (\rho E+p) v & \rho v \lambda_{I} & \rho v \lambda_{R}
\end{array}\right]^{T}, \\
& S=\left[\begin{array}{llllll}
0 & 0 & 0 & 0 & \rho \omega_{I} & \rho \omega_{R}
\end{array}\right]^{T},
\end{aligned}
$$


and a coordinate transformation from $(x, y)$ to $(\xi, \eta)$ is processed with the following grid metric:

$J^{-1}=\frac{\partial(x, y)}{\partial(\xi, \eta)}, \xi_{x}=J \cdot y_{\eta}, \xi_{y}=-J \cdot x_{\eta}, \eta_{x}=-J \cdot y_{\xi}, \eta_{y}=J \cdot x_{\xi}$.

The variables $\rho, u, v, E$, and $p$ are the density, x-direction velocity, $\mathrm{y}$-direction velocity, total energy per mass, and pressure, respectively. The equation of state can be expressed as

$p=(\gamma-1) \rho\left[E-\frac{u^{2}+v^{2}}{2}+\lambda_{R} Q\right]$,

where $Q$ is the total energy released during the chemical reaction.

According to numerical investigation [42], the processes of detonation initiation in hydrogen-air mixtures using both simplified and detailed chemical models are similar, and quite a few numerical studies on the flow configurations of ODWs adopted simplified (one-step or two-step) chemical reaction model [29,30,32,33, 35,43]; thus, a two-step chemical reaction model [44] is selected here to balance the computational cost and numerical accuracy. In the two-step chemical reaction model, $\lambda_{I}$ and $\lambda_{R}$ denote progress variables for the induction zone and the exothermic reaction zone, respectively, and have values between 0 and $1 . \omega_{I}$ and $\omega_{R}$ are the chemical reaction ratios, and their expressions are

$$
\begin{aligned}
& \omega_{I}=-K_{I} \mathrm{H}\left(\lambda_{I}\right) \exp \left(-\frac{E_{a}}{R T}\right), \\
& \omega_{R}=K_{R}\left[1-\mathrm{H}\left(\lambda_{I}\right)\right]\left(1-\lambda_{R}\right)^{v}, \\
& \mathrm{H}\left(\lambda_{I}\right)=\left\{\begin{array}{l}
1,0<\lambda_{I} \leq 1 \\
0, \lambda_{I} \leq 0
\end{array},\right.
\end{aligned}
$$

where $E_{a}$ is the activation energy; $v$ denotes the reaction order, which is typically $0.5 ; \mathrm{H}\left(\lambda_{I}\right)$ is the Heaviside function, which turns off the progress of $\lambda_{I}$ at the end of the induction zone; and $K_{I}$ and $K_{R}$ are pre-exponential factors used to control the induced reaction and the heat-releasing reaction, respectively.

All physical parameters used in the computation are normalized by referring to the uniform free stream, as follows (the symbol * denotes dimensional quantities, and the subscript $\infty$ indicates quantities under a free inflow stream):

$$
\begin{aligned}
& x=\frac{x^{*}}{l_{\text {ref }}}, y=\frac{y^{*}}{l_{\text {ref }}}, u=\frac{u^{*}}{\sqrt{p_{\infty} / \rho_{\infty}}}, v=\frac{v^{*}}{\sqrt{p_{\infty} / \rho_{\infty}}}, p=\frac{p^{*}}{p_{\infty}}, \\
& \rho=\frac{\rho^{*}}{\rho_{\infty}}, T=\frac{Q^{*}}{p_{\infty} /\left(R_{\infty} \rho_{\infty}\right)}, E=\frac{K_{I}^{*} l_{r e f}}{p_{\infty} / \rho_{\infty}}, Q=\frac{Q^{*}}{p_{\infty} / \rho_{\infty}}, \\
& E_{a}=\frac{E_{a}^{*}}{p_{\infty} / \rho_{\infty}}, K_{I}=\frac{t^{*} / \rho_{\infty}}{l_{\text {ref }} / \sqrt{p_{\infty} / \rho_{\infty}}}, \Psi=\frac{\Psi^{*}}{R_{\infty}},
\end{aligned}
$$

where $\Psi$ is a specific gas constant or specific heat, given here as representative, and $K_{R}=K_{I} K_{R}^{*} / K_{I}^{*}$.

To compute the inviscid fluxes, the weighted essentially nonoscillatory (WENO) [45] finite difference method is used to construct the left and right states. In this paper, the WENO-Z method is adopted to decrease numerical dissipation and improve the resolution efficiency of the WENO-JS scheme with the new smoothness indicators [46]. A Riemann problem solver, the Roe-HLLE method $[47,48]$, which is a low-dissipation and robust flux splitting method, is employed to obtain the numerical fluxes.

When the governing equations have been processed with space discretization, an ordinary differential equation is obtained. To achieve high-order accuracy and good stability simultaneously, the additive semi-implicit Runge-Kutta method implemented by ARKode in the SUNDIALS math library [49] is used to treat the

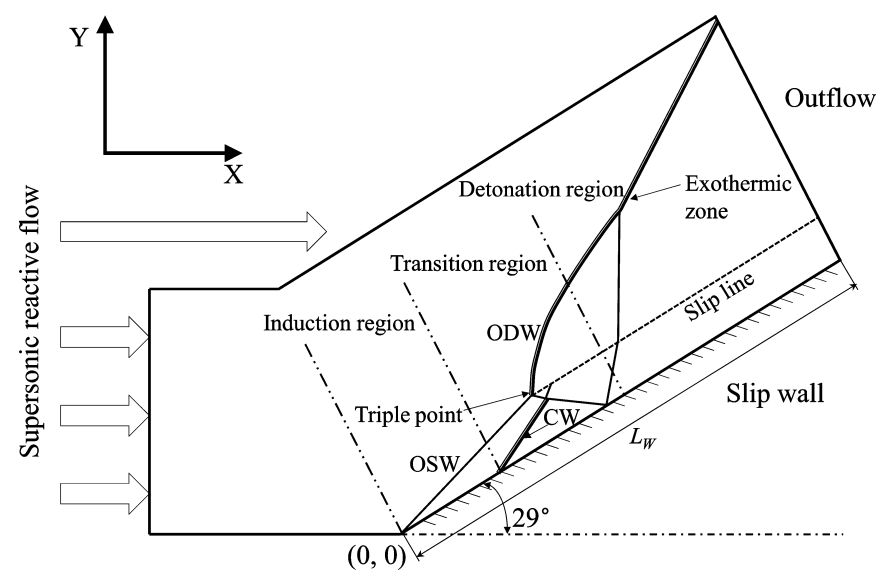

Fig. 1. Schematic depiction of the flow configuration and computational domain.

\begin{tabular}{l}
$\begin{array}{l}\text { Table } \mathbf{1} \\
\text { Physical-chemical parameters of mixtures. }\end{array}$ \\
\begin{tabular}{ll}
$M a_{\infty}$ & 7.0 \\
$p_{\infty}(\mathrm{Pa})$ & $1.0 \times 10^{5}$ \\
$T_{\infty}(\mathrm{K})$ & 300 \\
$R_{\infty}(\mathrm{J} /(\mathrm{kg} \cdot \mathrm{K}))$ & 397.57 \\
$R_{u}^{*}, R_{s}^{*}, R_{b}^{*}(\mathrm{~J} /(\mathrm{kg} \cdot \mathrm{K}))$ & $397.57,397.00,347.67$ \\
$\gamma_{u}, \gamma_{s}, \gamma_{b}$ & $1.40,1.32,1.16$ \\
$l_{r e f}(\mathrm{~m})$ & $2.5 \times 10^{-4}$ \\
\hline
\end{tabular} \\
\hline
\end{tabular}

stiffness problem in detonation physics computation. The butcher table used here is ARK4(3)6L[2]SA, which can achieve fourth-order accuracy in temporal discretization [50]. Additionally, the message passing interface (MPI) standard for message passing (as implemented in the MPICH library [51]) is used in the numerical simulations to improve the computational efficiency.

\subsection{Physical model and flow conditions}

A schematic depiction of the computational domain in this study is presented in Fig. 1. The free stream is parallel to the horizontal direction, and the angle of the wedge is fixed at $29 \mathrm{deg}$. The north and west boundaries are supersonic inflows, and the east boundary is a zeroth-order extrapolation outflow. The wedge surface is a slip wall boundary condition. The entire flow field illustrated in Fig. 1 is divided into three parts [21]: the induction region, the transition region and the detonation region.

The physical-chemical parameters of supersonic reactive flow based on hydrogen-air mixtures, are listed in Table 1 , and $Q$ is varied from 40.0 to 52.5 with $E_{a}=50.0$, similar to the parameters used by Leung and Radulescu [44]. In particular, to capture the main flow configuration, the length of the wedge $L_{W}$ is adjusted from 20 to $30 \mathrm{~mm}$ with increasing $Q$. Different specific heat ratios are considered to mimic the changes in mixtures from the unburned state to the post-shock state and finally to the burned state. The specific heats $c_{p}$ and $c_{v}$ and specific gas constant $R$ of the mixtures, labeled $\Psi$, are a function of the progress variables as follows:

$\Psi\left(\lambda_{I}, \lambda_{R}\right)=\Psi_{u} \lambda_{I}+\Psi_{s}\left(1-\lambda_{I}-\lambda_{R}\right)+\Psi_{b} \lambda_{R}$,

and the specific heat ratio $\gamma$ is calculated as:

$\gamma=\frac{c_{p}\left(\lambda_{I}, \lambda_{R}\right)}{c_{v}\left(\lambda_{I}, \lambda_{R}\right)}$ 

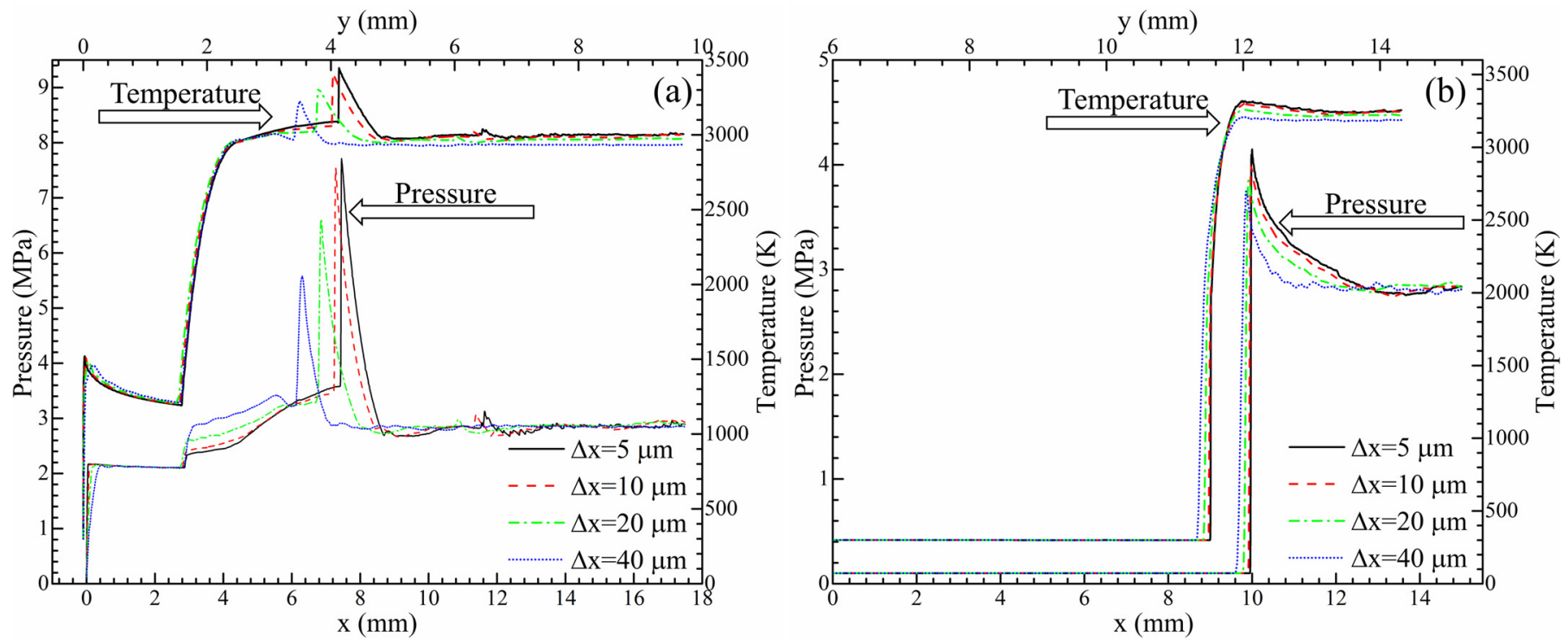

Fig. 2. Pressure $x$-profiles and temperature y-profiles along the wedge surface (a) and the line paralleled to the wall (b) for $Q=45.0$.

\subsection{Grid convergence}

The grid dependency of the numerical results in this study is evaluated for $Q=45.0$. Four grid sizes, i.e., $5,10,20$, and $40 \mu \mathrm{m}$ $(72,36,18$, and 9 grid cells in the reaction zone, respectively), are employed. The pressure and temperature profiles along the wedge direction are depicted in Fig. 2. The simulation results show that the key features of the flow fields could be captured with all utilized grid sizes; however, the fine grid achieves a good resolution of the shear layer and vortex. The pressure distribution versus the $\mathrm{x}$ coordinate and the temperature distribution versus the $\mathrm{y}$ coordinate along the wedge surface are given to clarify the results (see Fig. 2 (a)). Both the height and the location of the pressure and temperature peaks are similar for grid sizes of 5 and $10 \mu \mathrm{m}$. Fig. 2 (b) shows the distribution along the line that started at point $(0$, 6) and continued parallel to the wedge surface; a comparable tendency to that shown in Fig. 2 (a) can also be found. Therefore, the grid resolution studies indicate that a grid size of $\Delta x=10 \mu \mathrm{m}$ can resolve the flow field and guarantee reliable numerical data; thus, this size is adopted in the following numerical studies.

\subsection{Wave angle and shock polar analysis}

As a particle crosses a shock wave, the shock polar diagrams can provide the locus of all post-shock flow states, which is useful in the analysis of shock interaction flows. The Rankine-Hugoniot relations considering the chemical energy released in a thin reaction zone following the shock wave can be obtained. The wave angle-deflection angle $\beta-\theta$ and pressure ratio-deflection angle $\Phi-\theta$ are expressed as [52,53]

$$
\begin{gathered}
\tan \theta=\frac{-\gamma_{2}+\bar{\Omega} \pm \sqrt{\Omega}}{\gamma_{1}\left(\gamma_{2}+1\right) M a_{1}^{2}+\gamma_{2}-\bar{\Omega} \mp \sqrt{\Omega}} \cot \beta \\
\left(\text { denoted as }: \beta=\beta\left(\theta, M a_{1}, \gamma_{1}, \gamma_{2}, Q\right)\right), \\
\tan \theta=\frac{\Phi-1}{\gamma_{1} M a_{1}^{2}-(\Phi-1)} \sqrt{\Gamma-1} \\
\left(\text { denoted as }: \Phi=\Phi\left(\theta, M a_{1}, \gamma_{1}, \gamma_{2}, Q\right)\right),
\end{gathered}
$$

where the subscripts 1 and 2 denote the physical parameters before and after the ODW, respectively, $Q$ is the dimensionless chemical energy, and the symbol ' \pm ' in Eq. (9) is used to determine the overdriven and underdriven ODWs. The relation is reduced to the usual OSW relation if $Q=0$ and $\gamma_{1}=\gamma_{2}$. All polar curves in the following section are simply called shock polar curves regardless of whether $Q$ is equal to zero. The expressions for $\bar{\Omega}, \Omega, \Gamma$ are given as follows:

$$
\begin{aligned}
& \bar{\Omega}=\gamma_{1} M a_{1}^{2} \sin ^{2}(\beta), \\
& \Omega=\gamma_{2}^{2}+\bar{\Omega}^{2}-2 \gamma_{1} M a_{1}^{2} \sin ^{2}(\beta)\left[\frac{\gamma_{2}^{2}-\gamma_{1}}{\gamma_{1}-1}+\left(\gamma_{2}^{2}-1\right) Q\right], \\
& \Gamma=\frac{2 \gamma_{1} M a_{1}^{2}}{\left(\gamma_{1}-1\right)(\Phi-1)} \frac{\gamma_{1}-\gamma_{2}+\left(\gamma_{1}-1\right)\left[\Phi-1-\left(\gamma_{2}-1\right) Q\right]}{\left(\gamma_{2}+1\right)(\Phi-1)+2 \gamma_{2}} .
\end{aligned}
$$

As illustrated in Fig. 3, the physical meaning of the polar curves for an ODW is similar to that for an inert OSW, such as weak and strong solution branches, except that the curves exhibit a pressure jump from the initial state (point $A_{0}$ in Fig. 3 (b)) to the weak solution of $\theta=0$ (points $A_{1} \sim A_{4}$ in Fig. 3 (b)) because of the heat release. Additionally, the locus of CJ ODW can be obtained using the definition that the normal Ma behind the ODW is unity [54]. Clearly, it can be concluded that the shock polar curves (both $\beta-\theta$ and $\Phi-\theta$ ) shrink with an increasing amount of heat release; and an overdriven oblique detonation would be acquired for $\theta=29$ deg in the current work. Using the $\beta-\theta$ and $\Phi-\theta$ diagrams, we cannot provide quantitative insight or an exact description of the shock/combustion interaction because the structure of the reaction zone cannot be modeled approximately; in addition, the curvature effects of the combustion wave and shock wave strongly influence the flow configuration, which is difficult to quantify. Nevertheless, this helps us to qualitatively understand the shock/combustion interaction. Here, we define that positive angles in the shock polar curves correspond to clockwise deflections.

\section{Results and discussion}

Edney [40] classified six different shock/shock interaction flow patterns, i.e., Type I through VI interactions, based on an experimental study of OSWs impinging on blunt bodies. These categories have become the foundation for classifying shock/shock interactions and are also used as the guidelines for shock/combustion interactions here. When the strength of the impinging shock and the location of the intersection point relative to the bow shock are 

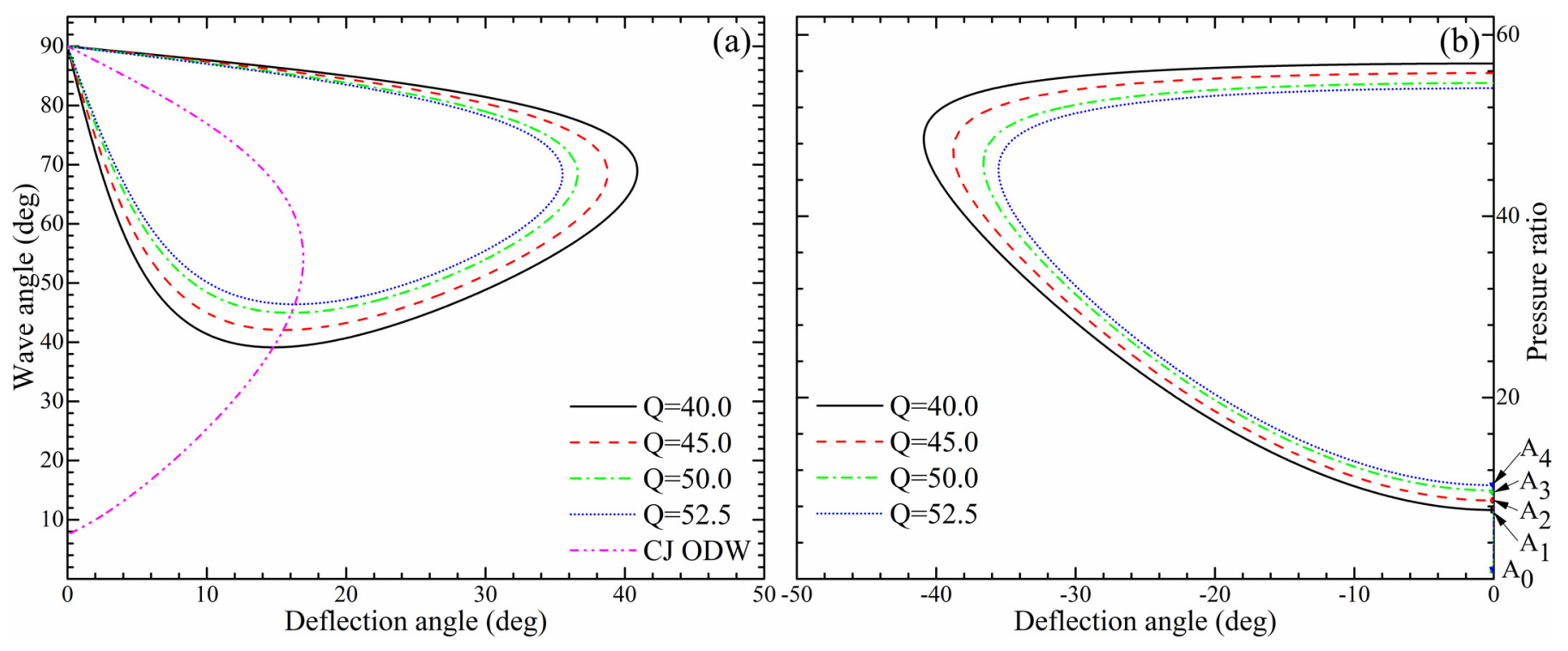

Fig. 3. Wave angle (a) and pressure ratio (b) versus deflection angle for various amounts of heat release.

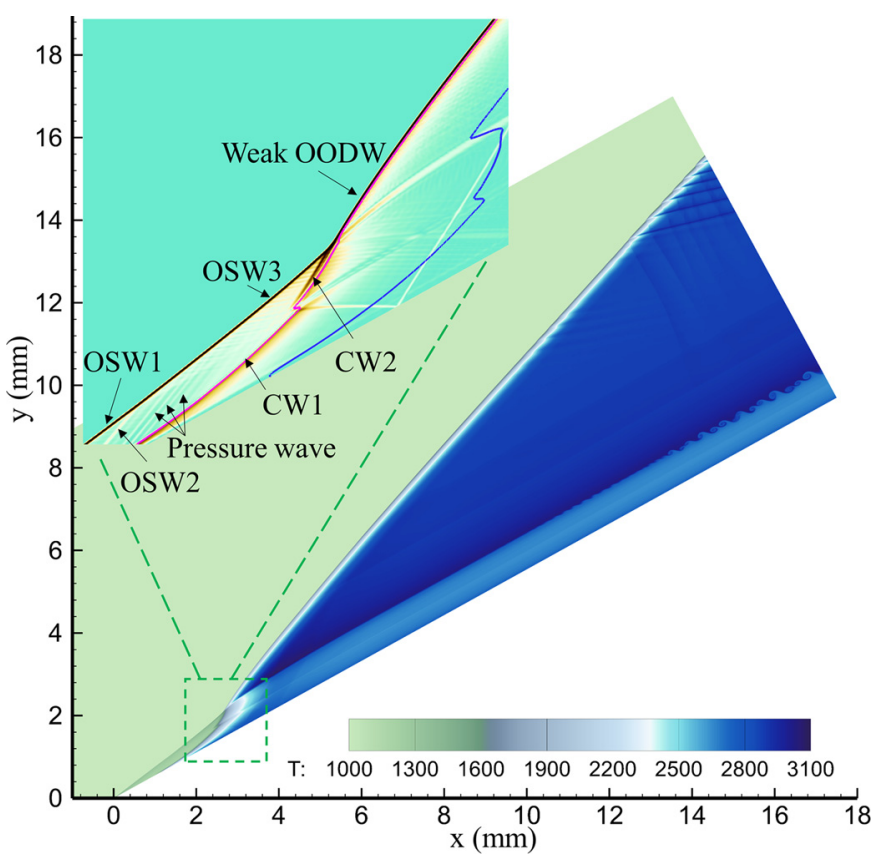

Fig. 4. Temperature contours and numerical schlieren for $Q=40.0$ (temperature unit: $\mathrm{K}$; solid lines: purple, product mass fraction $\lambda_{R}=0.05$; blue, product mass fraction $\lambda_{R}=0.95$ ). (For interpretation of the colors in the figure(s), the reader is referred to the web version of this article.)

altered at the typical shock/shock interference, transitions between different flow structures occur [53,55-57]. In the present study, the wave configurations of the transition region in the wedge-induced ODW, especially the interaction among inert OSW, CW and ODW, and the transitions between different shock/combustion interaction types, were investigated.

\subsection{Shock/combustion interaction with different amounts of heat release}

\subsubsection{Type VI interaction}

The temperature contours in the computational domain and the close-up numerical schlieren images in the transition region for $Q=40.0$ are plotted in Fig. 4 . The flow structure of the triple point connecting OSW3, CW2 and the weak overdriven ODW (OODW) is a Type VI interaction and is purely supersonic, and an expansion fan emanating from the triple point to divide the flow pattern. The combustion waves (CW1 and CW2) play a similar role as the shock wave emanating from the second wedge in the classic double wedge shock/shock interaction but produce a complex flow pattern. Because of the compression and disturbance produced by the combustion front, a series of pressure waves assemble and result in the formation of OSW2, which interacts with OSW1. Then, OSW3 is produced stronger than OSW1 and provides feedback to the downstream CW. The induced process that the reactants undergo when particles pass through OSW3 is different from that when they pass through OSW1, OSW2 and pressure waves, e.g., the temperatures at the end location of the induction zone are approximately 1325 $\mathrm{K}$ and $1200 \mathrm{~K}$, respectively. Therefore, an exothermic reaction is promptly produced, which results in CW2 following OSW3 traveling forward, called a triangle-convex combustion front.

The shock polar diagram and a schematic depiction of the flow configuration for $Q=40.0$ are given in Fig. 5, where the numbered regions in Fig. 5 (b) usually correspond to the numbered points in the shock polar diagram in Fig. 5 (a), and the pressure ratio is normalized by the free-stream pressure. It can be confirmed that particles in Regions (1), (3) and ( $\left.3^{\prime}\right)$ do not undergo exothermic reactions based on the contour line of the production mass fraction displayed in Fig. 4; thus, it can be assumed that these regions are uniform flow fields even though the pressure waves in Region (3) contribute to a small but negligible pressure jump. However, Regions (2) and (4)-(6) experience continuous changes due to chemical energy release. In particular, for the weak OODW (curved PD in Fig. 5 (b)), a reaction zone with a large length-scale following the leading shock wave (see Fig. 4), polar $R_{2}(0.2 Q)$ and $R_{2^{\prime}}(Q)$ are given to depict the states after the PD with two exothermic progress variables. It should be noted that the shock polar is suitable only in the small region near the intersection point for curved shock/combustion waves.

Across shock IQ, Point (1) is located based on the requirements of the free-stream condition and wedge angle, and polar $\mathrm{R}_{3}$ emanating from Point (1) can be drawn. Then, Points (3) and $\left(3^{\prime}\right)$ are determined by the interaction between polar $R_{1}$ and $R_{3}$. However, it is difficult to exactly describe the entire non-uniform flow behind combustion $\mathrm{BC}, \mathrm{PC}^{\prime}$ and $\mathrm{FC}$, and thus, the state of the local zone in the flow field can only be roughly illustrated. The deflection angle of combustion BC would be calculated with the numerical results and cannot be theoretically determined from the shock 


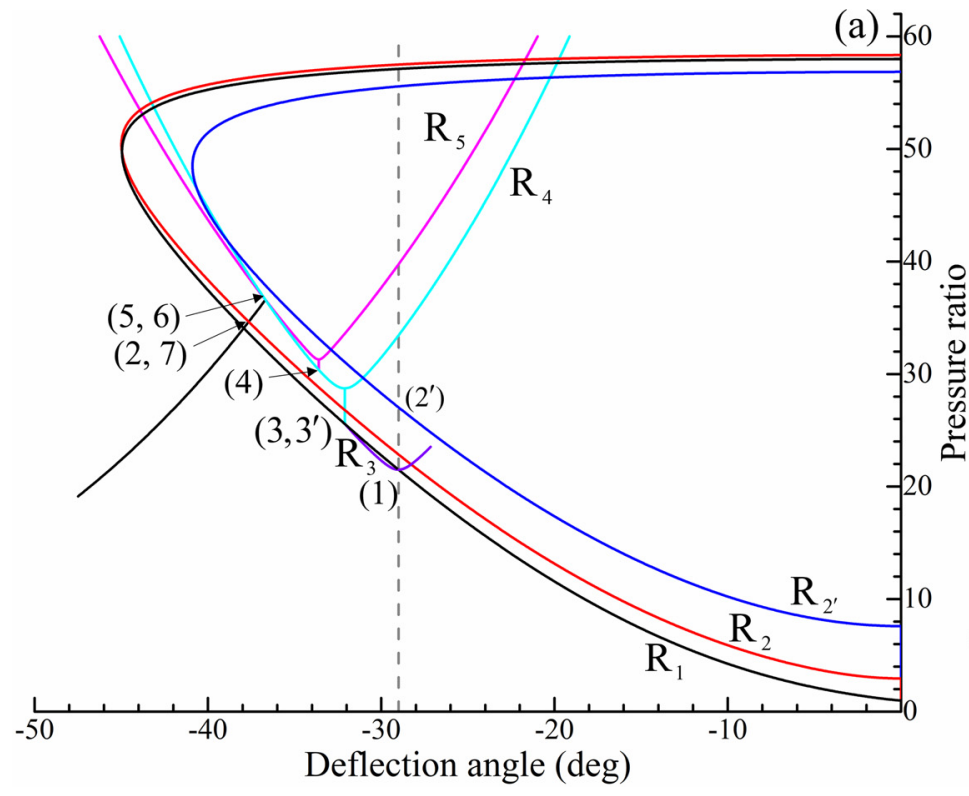

(b)

Fig. 5. Shock polar diagram (a) and schematic diagram (b) of Type VI interaction for $Q=40.0$.

polar diagram. Then, Point (4) and polar $\mathrm{R}_{5}$ can be obtained. Next, the state behind $\mathrm{PC}^{\prime}$, e.g., Points (5) and (6), is co-located at the interaction between polar $R_{4}$ and $R_{5}$. Regions (6) and (2) must be connected by an expansion wave fan since the pressure in Region (6) is larger than that in Region (2). Therefore, Points (2) and (7), at a pressure ratio of 34.6 , lie on the interaction between polar $R_{2}$ and the expansion path originating from Point (6), which compares well with the simulation value of 33.3. From Region (2) to Region $\left(2^{\prime}\right)$, shock polar sets bridge two points with a continuous release of heat. The pressure ratio of Point $\left(2^{\prime}\right)$ is determined to be 27.0 from the shock polar analysis and 28.3 from the numerical results.

According to the above discussions, certain conditions should be considered if the heat release increases. When Point (6) lies below polar $\mathrm{R}_{2}$, a shock, rather than an expansion fan, connects the state with Point (2). Once Point (6) moves up and to the left in the diagram, neither the expansion path nor the shock polar can intersect $R_{2}$ or $R_{2^{\prime}}$ below the sonic point; thus, a pure supersonic flow would not be maintained. A related simulation study is conducted in the following section.

\subsubsection{Type Vinteraction}

The numerical results are illustrated in Fig. 6 for increasing amounts of heat release $(Q=45.0$ and 50.0). Fig. 6 (a) shows that the flow structure between CW1 and OSW1 in the transition region is similar to that for $Q=40.0$. However, the interaction near the upper triple point (UTP) is entirely different. The wave configuration for $Q=45.0$ satisfies a key characteristic, allowing it to be classified as a Type $\mathrm{V}$ interaction. The ODW emanating from the UTP is curved to match the neighboring high pressure, yielding a strong OODW, which is not identical to the weak solution for $Q=40.0$. The strong OODW is affected by the disturbance downstream since a following subsonic zone, which would be attenuated successively, and transforms into a weak OODW based on the value of Ma (see the sonic line in Fig. 6 (a)). It should be emphasized that the transverse wave (TW) emitting from the UTP is an inert shock, and the chemical energy is liberated in the following front instead (see the solid purple line in Fig. 6 (a)). The instabilities of the vortex structure are more intense than those for $Q=40.0$, and the Kelvin-Helmholtz $(\mathrm{KH})$ instability along the slip line is clearly visible.

Similar computational results for $Q=50.0$ are plotted in Fig. 6 (b). The flow pattern is also a Type V interaction; however, some structures are dissimilar to those in Fig. 6 (a). Obviously, the shock wave impinging on the wedge surface undergoes Mach reflection. CW3 (see Fig. 6 (b)) in this case is not emitted from the middle triple point (MTP), but rather from a certain upstream position along the TW. It is possible that the UTP and MTP are located in the exothermic reaction zone, and the wave pattern is controlled by both fluid dynamics and chemical non-equilibrium effects. To match the flow field around the MTP, a knot structure, instead of a simple multipoint structure, should be established in this zone. In addition, a triangle-convex combustion front (which consists of CW1 and CW2, as shown in Fig. 6 (a)) disappears because the longer induction region and the shorter transition region lead to a lack of interaction between OSW1 and OSW2.

As a representative case of a Type $\mathrm{V}$ interaction, the shock polar and flow field diagrams for $Q=50.0$ are depicted in Fig. 7. The determination of shock polar $R_{1}$ and $R_{3}$ and Points (1) and (3) is analogous to that in Fig. 5 (a); however, the polar for the ODW is characterized only by $R_{2}$ due to the narrow reaction strip shown in Fig. 6 (b). In this case, it is not possible to bridge the gap between Region (4) and a weak solution branch of polar $R_{2}$ via shock waves or expansion waves. Another flow pattern is required to have the states meet behind detonation PD and combustion BC. Polar $\mathrm{R}_{7}$ originates from Point (3) and is used to describe the post-shock state of shock PC, and Region (2) should be determined using the interaction between polar $R_{2}$ and $R_{7}$ since Regions (2) and (7) are separated by a contact discontinuity. The pressure value of Points (2) and (7) in the shock polar diagram is 53.9, which is slightly smaller than the numerical value of 58.1.

The state behind combustion BC should be determined for different conditions because of the non-uniform flow. Region (4) near the MTP can be obtained with polar $\mathrm{R}_{4}$ and the given deflection angle, as shown in Fig. 7 (a). Then, Points (5) and (6) are determined by the intersection of polar $R_{5}$ originating from Point (4) and polar $R_{6}$ originating from Point (7), which are below the sonic point of the corresponding polar. The Mach reflection configuration GH can be determined by a typical shock/shock interaction because all reactants have been transformed into products, and no chemical energy is liberated in this region. However, it is difficult to achieve the state of Region $\left(4^{\prime}\right)$ while having to match the physical wall boundary, where the streamlines of Region $\left(4^{\prime}\right)$ are parallel to the wedge surface. According to the numerical results, a continuous increase in pressure exists between Region (4) and 

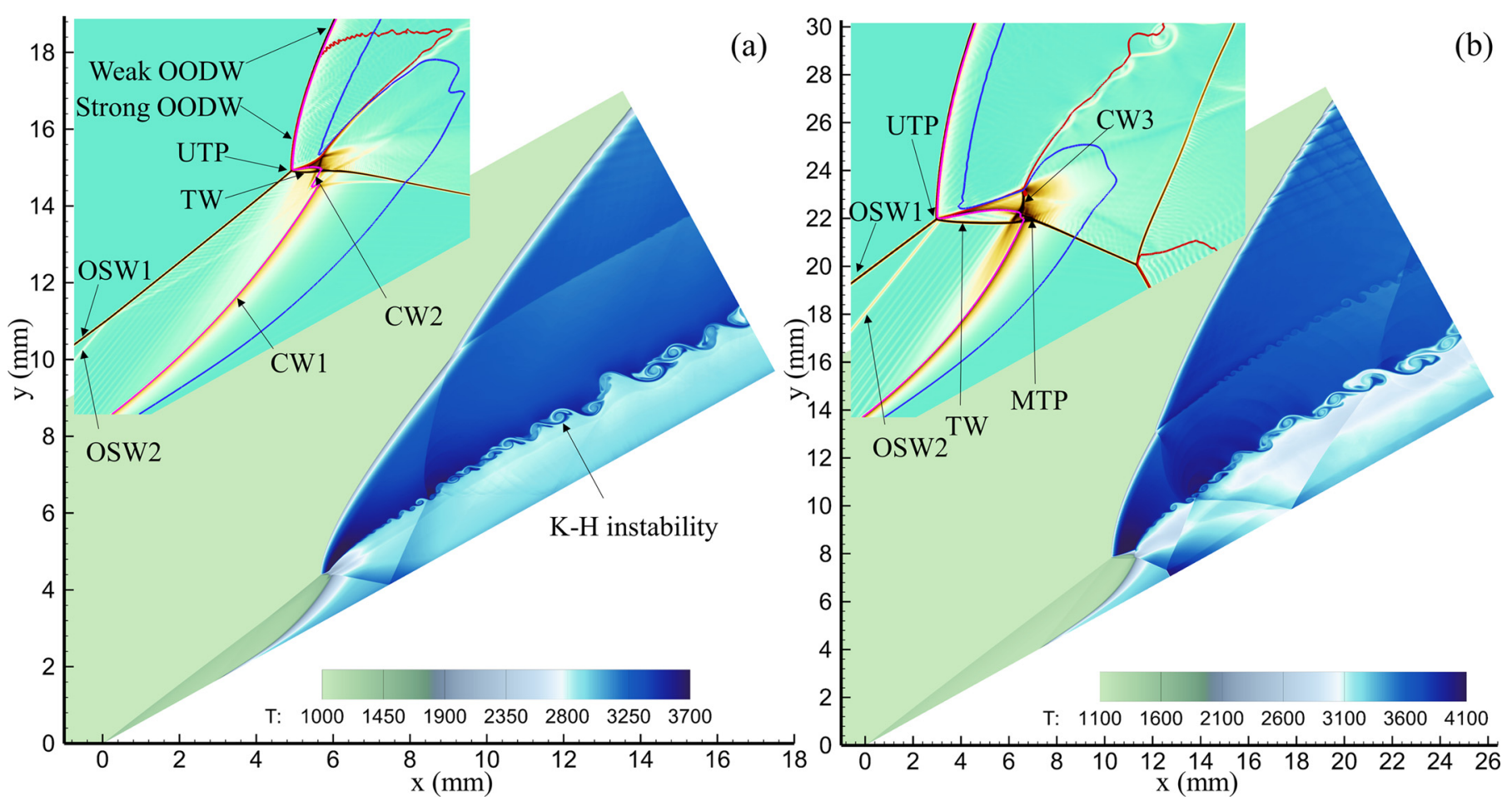

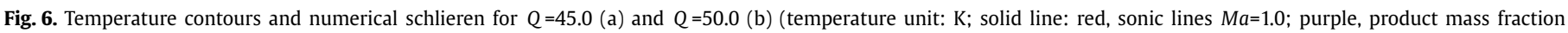
$\lambda_{R}=0.05$; blue, product mass fraction $\lambda_{R}=0.95$ ).
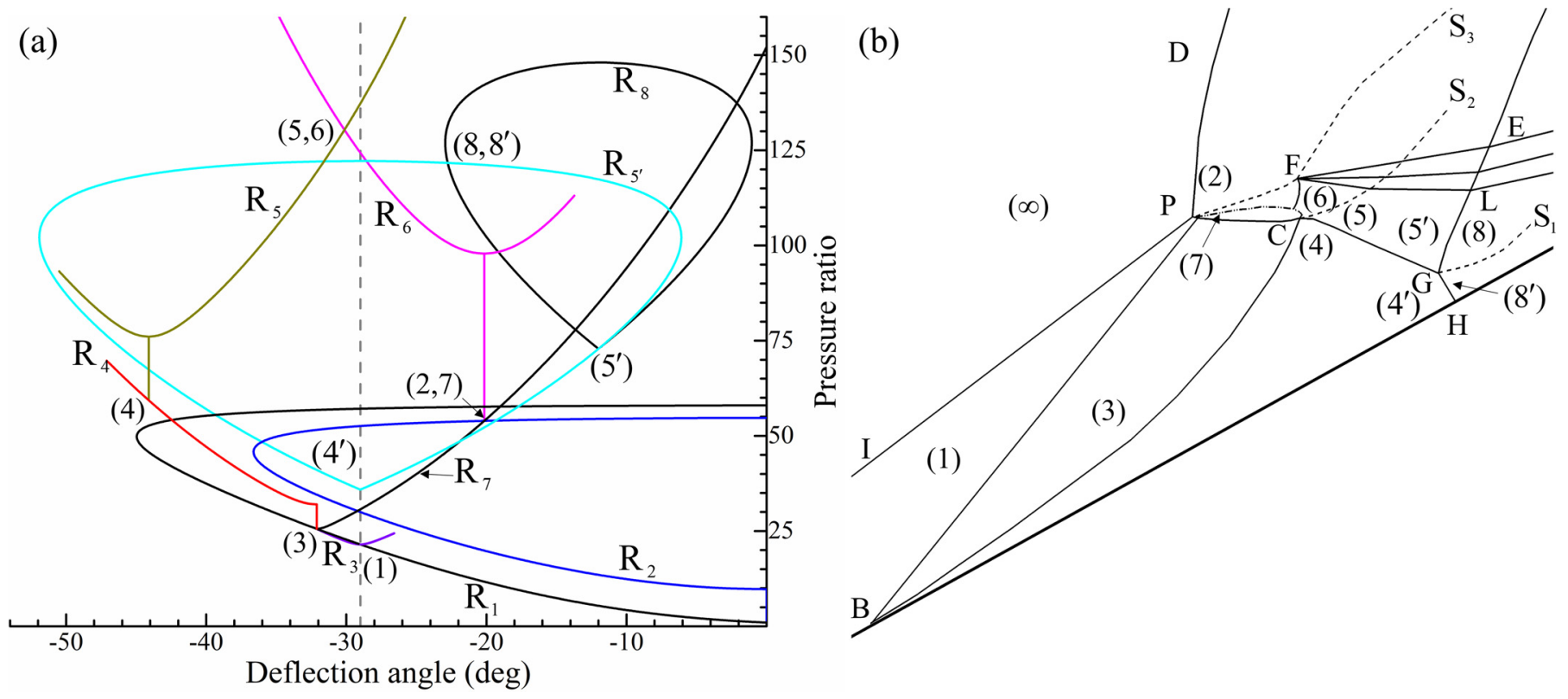

Fig. 7. Shock polar diagram (a) and schematic diagram (b) of Type V interaction for $Q=50.0$.

Region $\left(4^{\prime}\right)$ because of the curved combustion BC. The streamlines of Region $\left(4^{\prime}\right)$ turn back and then become parallel to the wall due to the neighboring high-pressure zone. Illustrating this process in the shock polar diagram is complicated and arduous; thus, the numerical value is employed to represent Point $\left(4^{\prime}\right)$. Then, Points (8) and $\left(8^{\prime}\right)$ are co-located with the interaction of $R_{5^{\prime}}$ emanating from Point $\left(4^{\prime}\right)$ and polar $R_{8}$.

\subsubsection{Type IV interaction}

For $Q=52.5$, a time sequence of numerical results is illustrated in Fig. 8 to explain the evolution process from the initiation of the ODW to the quasi-steady state flow. As shown in Fig. 8 (a), two remarkable Mach stems with the following subsonic area can be found. The flow configuration is unsteady, which facilitates the detonation front and Mach stems shifting upstream, due to the exothermic reaction and the pressure disturbance in the transition region. Then, the deflection angle of the TW emanating from the UTP decreases, which incurs the tendency of regular reflection on the MTP (see Fig. 8 (b)). Furthermore, the lower Mach stem (LMS) on the wedge surface grows, moves toward the MTP and eventually interacts with the MTP (as shown in Fig. 8 (c)). The two distinguishable Mach stems in Fig. 8 (d), which are reformed but 

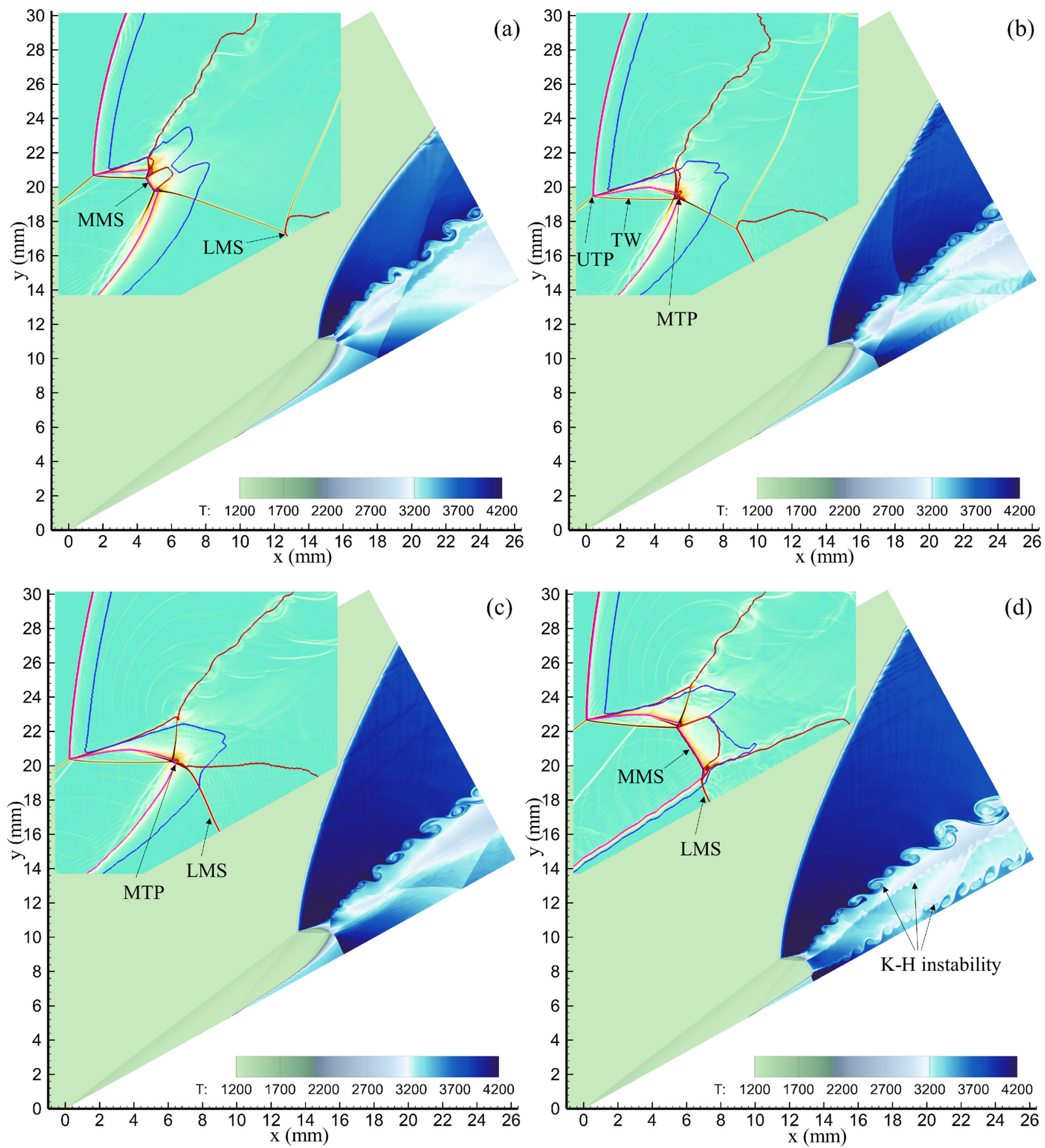

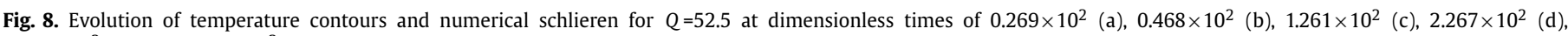
$4.225 \times 10^{2}$ (e) and $6.392 \times 10^{2}$ (f) (temperature unit: K; solid lines: red, sonic line $M a=1.0$; purple, reactant mass fraction $\lambda_{R}=0.05$; blue, product mass fraction $\lambda_{R}=0.95$ ).

not the same as those in Fig. 8 (a), are connected by a short sinuous shock wave. In addition to the slipstream originating from the UTP, KH instability phenomena appear along the shear layers behind both the middle Mach stem (MMS) and the LMS. The LMS disappears at the terminal location of the induced region, and the MMS progresses upstream and begins to shorten. In particular, a pressure wave emerges downstream (see Fig. 8 (e)). As shown in Fig. 8 (f), the Mach stem eventually remains at a position that is $4.37 \mathrm{~mm}$ from the apex of the wedge, and the moving shock wave downstream interacts with the ODW front. It is well known that the ODW would not be influenced by the post-shock supersonic flow; however, a pressure wave established downstream has an effect on the ODW front here, which provides another physical phenomenon that requires further study. The wave configuration of the transition region shown in Fig. 8 (f) can fit a Type IV interaction. 

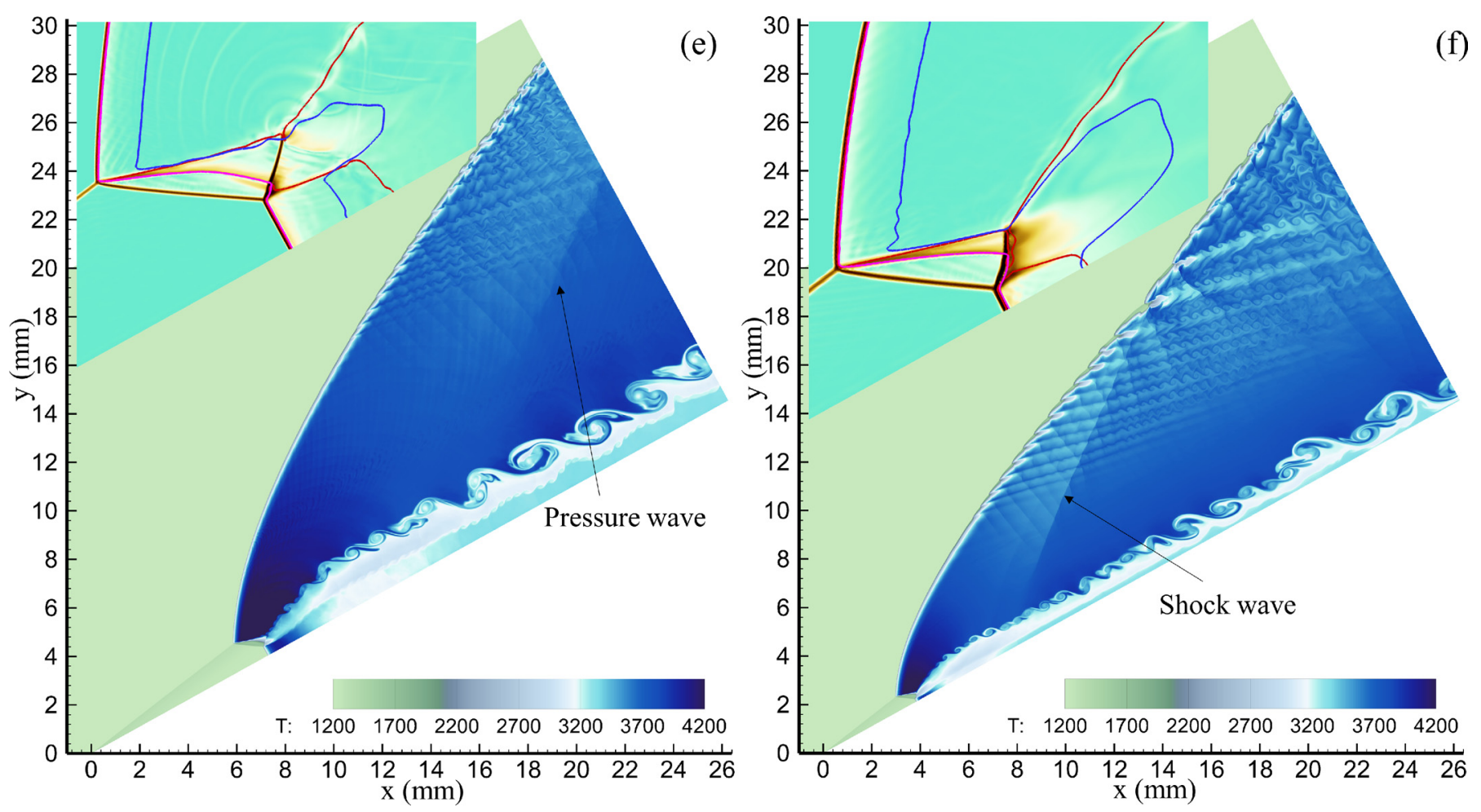

Fig. 8. (continued)

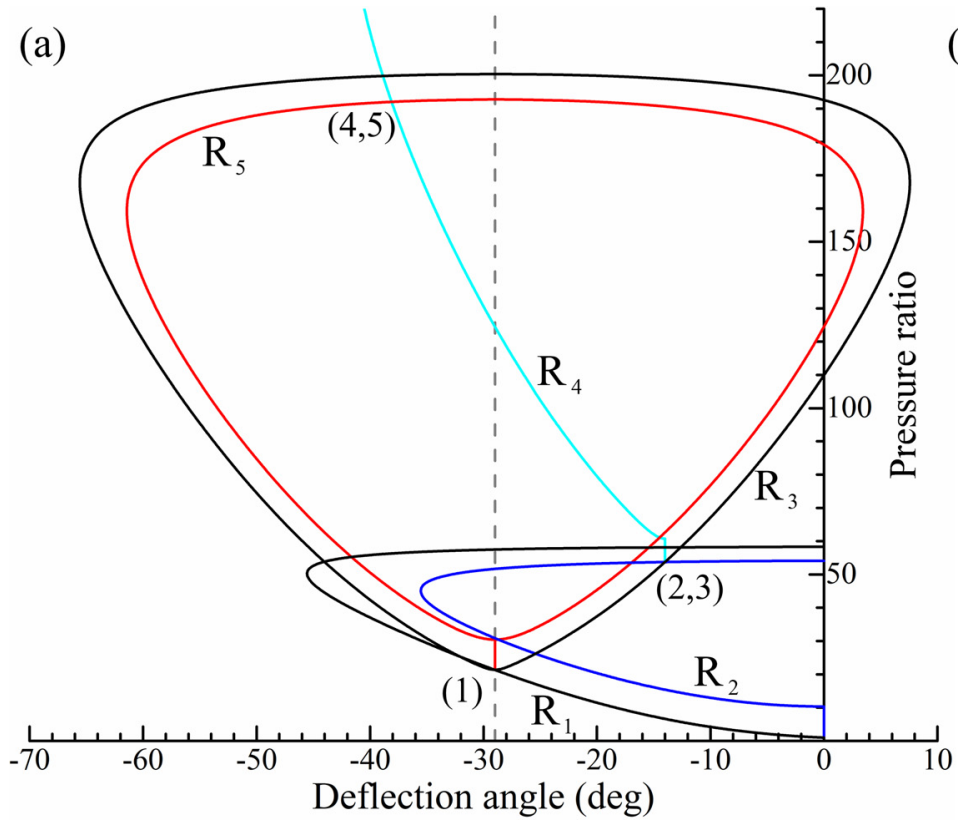

(b)

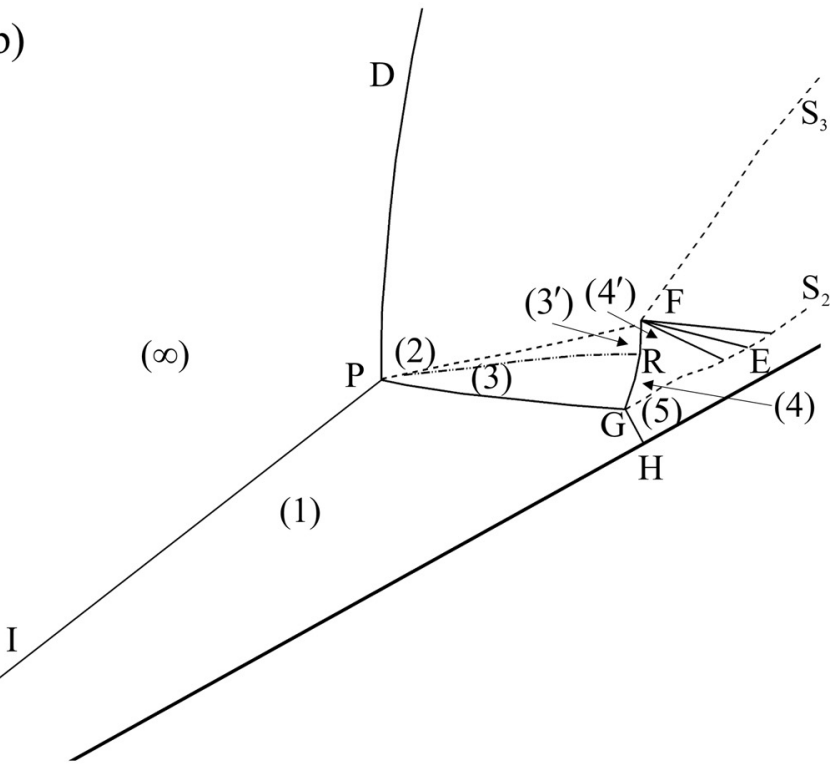

Fig. 9. Shock polar diagram (a) and schematic diagram (b) of Type IV interaction for $Q=52.5$.

Fig. 9 plots the shock polar and schematic diagrams for Fig. 8 (f). Likewise, regarding the state behind detonation PD, Points (2) and (3) are acquired from the intersection between polar $R_{2}$ and $\mathrm{R}_{3}$ originating from Point (1) without heat release. Then, polar $\mathrm{R}_{4}$ originating from Point (3) can be drawn. The shock PG impinging on the wedge surface undergoes an irregular reflection, and the states, e.g., Points (4) and (5), can be acquired based on the intersection of polar $R_{4}$ and $R_{5}$ originating from Point (1) with heat release. Here, the irregular reflection is an inverse Mach reflection that would lead to a diverging stream tube that cannot match the subsonic zone behind the Mach stem with the supersonic flow downstream; this type of wave configuration is theoretically impossible [57]. However, additional physical conditions imposed downstream of the interaction could stabilize such a theoretically unstable wave pattern. Due to the existence of the exothermic front PR, the states before combustion GF are different, which leads to a local high-pressure zone $\left(4^{\prime}\right)$. Then, the diverging stream tube would be compressed and converge, accelerating the subsonic flow to sonic flow, as shown in Fig. 10. Finally, a diverging-converging-diverging stream tube is formed to satisfy the supersonic flow condition downstream. 


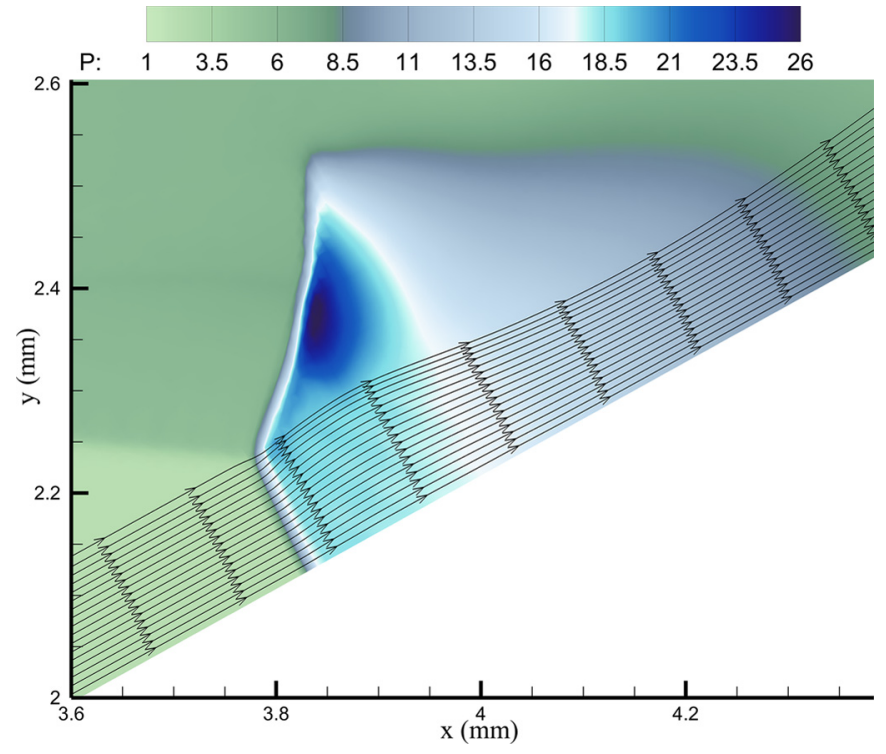

Fig. 10. Close-up images of pressure contours near the Mach reflection at dimensionless time $6.392 \times 10^{2}$ for $Q=52.5$ (pressure unit: $\mathrm{MPa}$ ).

\subsection{Transition principle}

Unlike the prediction of the transition criterion of the shock/ shock interaction, it is arduous to propose an accurate critical condition analytically for Type VI to Type V transition. Thus, a qualitative discussion is given based on extended numerical simulations. The computed results show that the transition path is Type $\mathrm{VI} \rightarrow \mathrm{I} \rightarrow \mathrm{V}$, and the transition process is continuous for the given range of heat release.

Zoomed-in numerical shadowgraphs of the transition region for different amounts of heat release are given in Fig. 11 and can describe the transition from Type VI to Type V. The flow configuration for $Q=41.5$ shown in Fig. 11 (a) is analogous to that for $Q=40.0$ except that an OSW, rather than an expansion fan, emanates from the UTP. Essentially, the flow pattern shown in Fig. 11 (a) is Type I rather than Type VI. It is suggested that the pressure ratio behind the ODW near the triple point is higher when $Q$ increases, and a shock wave is established to match the highpressure condition. With further increases in $Q$, the structure of the reaction zone would be narrower, leading to a shrunken shock polar $R_{2}$ shown in Fig. 5 (a) with an intersection point at the strong solution branch with the polar originating from Point (6). Then, a subsonic zone emerges (see Fig. 11 (b)), which can be called a strong Type I interaction. When $Q=43.0$, Point (6) in Fig. 5 (a) moves up and to the left in the diagram, and the shock polar originating from Point (6) cannot intersect with polar $R_{2}$. Then, another flow pattern is required, a short TW emerges, and the subsonic zone grows, as exhibited in Fig. 11 (c). Finally, a Type V shock/combustion interaction can be clearly visualized for $Q=44.0$, as depicted in Fig. 11 (d). It should be emphasized that a direct Type $\mathrm{VI} \rightarrow \mathrm{V}$ transition, not a Type $\mathrm{VI} \rightarrow \mathrm{I} \rightarrow \mathrm{V}$ transition, occurs for the classic double-wedge shock/shock interaction with the first wedge angle of $29 \mathrm{deg}$ when the freestream Mach number is larger than the critical Mach number of 4.91. However, for the given $M a=7.0$ in the current work, the transition route of Type $\mathrm{VI} \rightarrow \mathrm{I} \rightarrow \mathrm{V}$ occurs for the shock/combustion interaction here inasmuch as the relative locations of Point (6) and polar $R_{2}$ in Fig. 5 (a) change when the amount of heat release increases.

To gain insight into the Type V to Type IV transition, a complementary computation is conducted with $Q$ ranging from 51.0 to 52.0 , and the numerical results are plotted in Fig. 12. The structures for $Q=51.0$ and 51.5, illustrated in Fig. 12 (a) and (b), re- spectively, are similar to that for $Q=50.0$, except that the LMS is lengthened. For $Q=51.8$, a new version of the Type $V$ interaction is found in Fig. 12 (c), where a Mach reflection occurs at the MTP, and a subtle but distinct subsonic zone can be seen behind the short MMS. Observing the numerical results for $Q$ varying from 50.0 to 51.8, a notable trend can be found: the LMS length increases from 0.31 to $0.75 \mathrm{~mm}$, whereas the distance between the LMS and the UTP along the wedge surface direction decreases from 0.84 to $0.55 \mathrm{~mm}$, which is similar to the evolution process described for $Q=52.5$. It can be concluded that the LMS would collide with the MTP/MMS, promoting a transition from Type $\mathrm{V}$ to Type IV interaction when $Q$ increases gradually (see Fig. 12 (d)).

When $Q$ increases, the numerical results show that the transition may result in significant changes in pressure and heating loads along the wedge surface, e.g., the peak pressure in the case of $Q=52.5$ is nearly 6 times that in the case of $Q=40.0$. Additionally, the transition from Type $\mathrm{V}$ to Type IV interaction makes the UTP and ODW front travel upstream along the wedge; this should be considered in the design of ODWEs and the control of the standing ODW.

\section{Conclusion}

A finite difference method solver adopting WENO-Z reconstruction and Roe-HLLE flux splitting in spatial discretization and the additive Runge-Kutta method in time-marching is used to investigate the shock/combustion interactions in the transition region of a wedge-induced oblique detonation wave (ODW). According to Edney's categorization, Type VI, I, V and IV interactions are observed computationally for dimensionless heat release $Q$ ranging from 40.0 to 52.5 .

When $Q=40.0$, Type VI interaction occurs, where an expansion fan emanating from the triple point. When $Q$ increases to 41.5 and 42.0, a Type I interaction occurs, and a shock wave is produced from the triple point instead. For both Type VI and Type I interaction, a triangle-convex combustion front is formed due to the interaction between the shock wave emanating from the wedge tip and the inert oblique shock wave produced from the combustion wave. When $Q$ is varied from 44.0 to 51.8 , three versions of Type $\mathrm{V}$ interaction are found numerically when considering the reflection patterns at the middle triple point (MTP) and the lower triple point (LTP). The three versions of Type $\mathrm{V}$ interaction are as follows: 1) a regular reflection occurs at both the MTP and the LTP; 2) a regular reflection occurs at the MTP, and a Mach reflection occurs at the LTP; and 3) a Mach reflection occurs at both the MTP and the LTP. When $Q$ is larger than 52.0, a Type IV interaction is produced, and an inverse Mach reflection is formed with a diverging-converging-diverging stream tube downstream of the Mach stem.

The transition principles of different shock/combustion interactions are given based on shock polar analyses and numerical simulations. The transition path of Type VI to Type V interaction is Type $\mathrm{VI} \rightarrow \mathrm{I} \rightarrow \mathrm{V}$. The reason for the Type $\mathrm{VI} \rightarrow \mathrm{I}$ transition is that the flow states behind the combustion wave are overexpanded with respect to the state behind the weak overdriven ODW. The Type $\mathrm{I} \rightarrow \mathrm{V}$ transition occurs as $Q$ increases because the overdriven ODW cannot match the flow field behind the combustion wave. Neither the expansion path nor the shock polar originating from the state behind the combustion wave can interact with the ODW polar. For Type V to Type IV transition, the Mach stem impinging on the wedge surface travels upstream and interacts with the MTP, which triggers the transition.

Although the critical heat release of the transition procedure can be obtained numerically, it is difficult to present a transition criterion for the shock/combustion interaction, which is controlled not only by hydrodynamics but also by chemical kinetics. A so- 

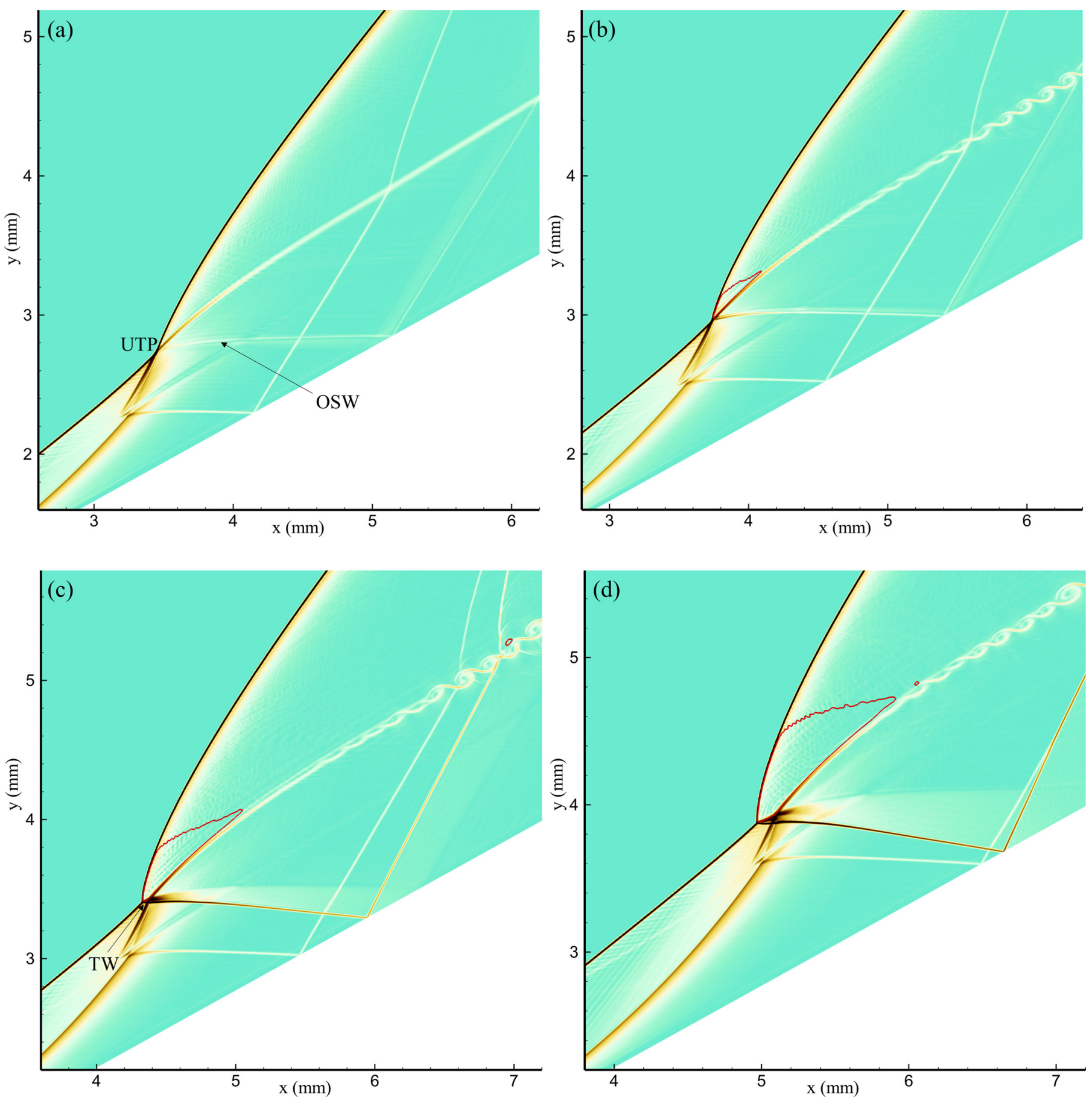

Fig. 11. Zoomed-in numerical schlieren of the transition region of ODW for $Q=41.5$ (a), $Q=42.0$ (b), $Q=43.0$ (c) and $Q=44.0$ (d) (solid red line: sonic line $M a=1.0$ ).

phisticated model that couples the flow and exothermic reactions is needed to treat the complex interaction structure, and additional investigations of the mechanism of the shock/combustion interaction should be performed in the future.

\section{Declaration of competing interest}

There is no competing interest.

\section{Acknowledgements}

The research is supported by the National Natural Science Foundation of China under grant numbers: 11672309 and 11472279 .

\section{References}

[1] J. Urzay, Supersonic combustion in air-breathing propulsion systems for hypersonic flight, Annu. Rev. Fluid Mech. 50 (2018) 593-627, https://doi.org/10.1146/ annurev-fluid-122316.

[2] K. Kailasanath, Review of propulsion applications of detonation waves, AIAA J. 38 (2000) 1698-1708, https://doi.org/10.2514/2.1156.

[3] P. Wolanski, Detonative propulsion, Proc. Combust. Inst. 34 (2013) 125-158, https://doi.org/10.1016/j.proci.2012.10.005.

[4] W. Huang, L. Yan, J. Tan, Survey on the mode transition technique in combined cycle propulsion systems, Aerosp. Sci. Technol. 39 (2014) 685-691, https://doi. org/10.1016/j.ast.2014.07.006.

[5] N.N. Smirnov, V.F. Nikitin, L.I. Stamov, E.V. Mikhalchenko, V.V. Tyurenkova, Rotating detonation in a ramjet engine three-dimensional modeling, Aerosp. Sci. Technol. 81 (2018) 213-224, https://doi.org/10.1016/j.ast.2018.08.003. 

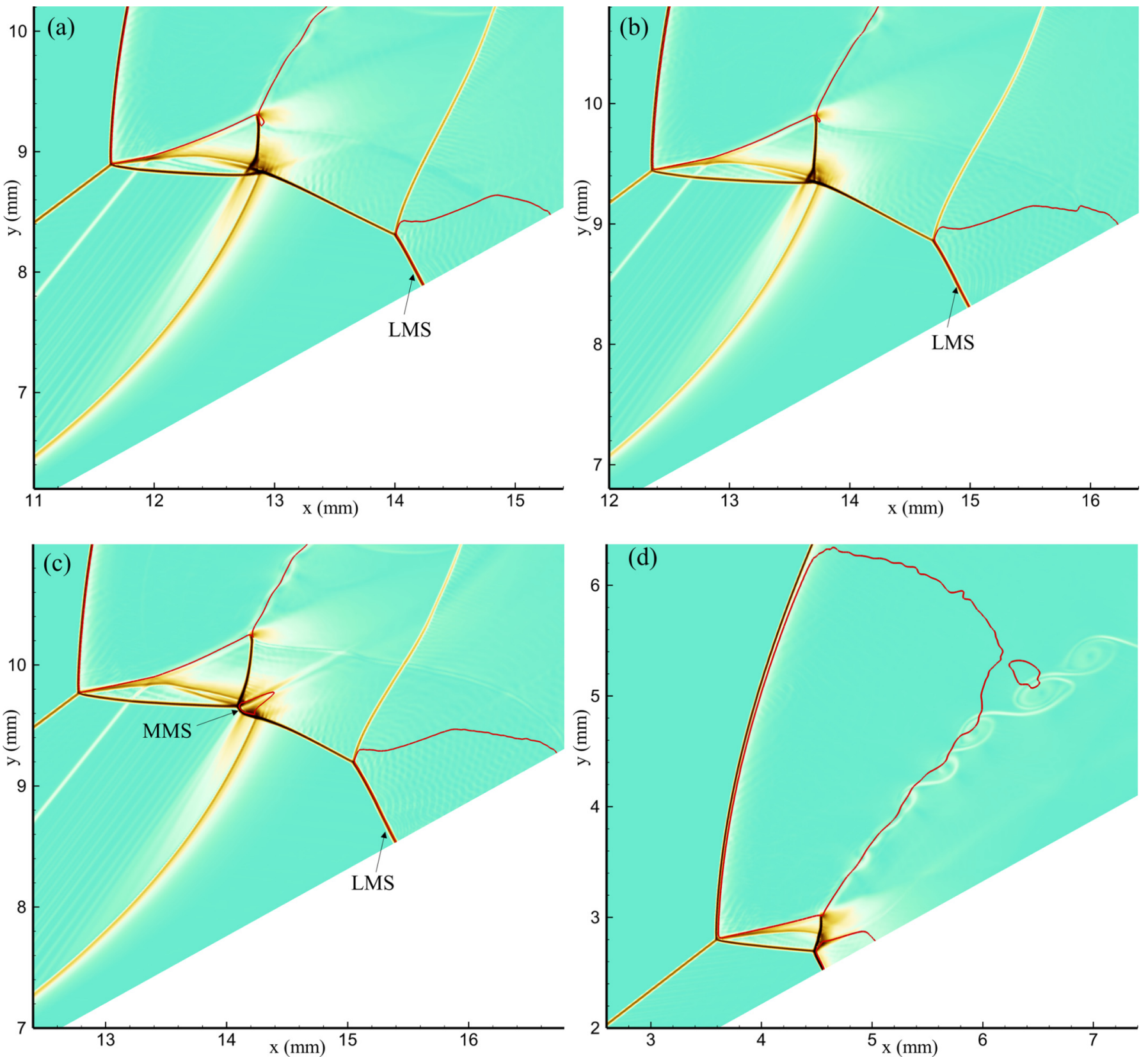

Fig. 12. Zoomed-in numerical schlieren of the transition region of ODW for $Q=51.0$ (a), $Q=51.5$ (b), $Q=51.8$ (c) and $Q=52.0$ (d) (solid red line: sonic line $M a=1.0$ ).

[6] S.M. Frolov, V.S. Aksenov, V.S. Ivanov, I.O. Shamshin, A.E. Zangiev, Air-breathing pulsed detonation thrust module: numerical simulations and firing tests, Aerosp. Sci. Technol. 89 (2019) 275-287, https://doi.org/10.1016/j.ast.2019.04. 005 .

[7] D.A. Schwer, K. Kailasanath, T. Kaemming, Pressure characteristics of a ram-RDE diffuser, Aerosp. Sci. Technol. 85 (2019) 187-198, https://doi.org/10.1016/j.ast. 2018.11.006.

[8] J.Z. Ma, S. Zhang, M. Luan, J. Wang, Experimental investigation on delay time phenomenon in rotating detonation engine, Aerosp. Sci. Technol. 88 (2019) 395-404, https://doi.org/10.1016/j.ast.2019.01.040.

[9] L.-F. Zhang, S.-J. Zhang, Z. Ma, M.-Y. Luan, J.-P. Wang, Three-dimensional numerical study on rotating detonation engines using reactive Navier-Stokes equations, Aerosp. Sci. Technol. 93 (2019) 105271, https://doi.org/10.1016/j.ast.2019. 07.004.

[10] X. Zhang, L. Yue, T. Huang, Q. Zhang, X. Zhang, Numerical investigation of mode transition and hysteresis in a cavity-based dual-mode scramjet combustor, Aerosp. Sci. Technol. 94 (2019) 105420, https://doi.org/10.1016/j.ast.2019. 105420.

[11] Z. Wang, M. Sun, H. Wang, J. Yu, J. Liang, F. Zhuang, Mixing-related low frequency oscillation of combustion in an ethylene-fueled supersonic combustor, Proc. Combust. Inst. 35 (2015) 2137-2144, https://doi.org/10.1016/j.proci.2014. 09.005 .
[12] M.-B. Sun, Z.-G. Wang, J.-H. Liang, H. Geng, Flame characteristics in supersonic combustor with hydrogen injection upstream of cavity flameholder, J. Propuls. Power 24 (2008) 688-696, https://doi.org/10.2514/1.34970.

[13] M. Sun, Z. Zhong, J. Liang, H. Wang, Experimental investigation on combustion performance of cavity-strut injection of supercritical kerosene in supersonic model combustor, Acta Astronaut. 127 (2016) 112-119, https://doi.org/10.1016/ j.actaastro.2016.05.035

[14] W. Huang, L. Yan, Numerical investigation on the ram-scram transition mechanism in a strut-based dual-mode scramjet combustor, Int. J. Hydrog. Energy 41 (2016) 4799-4807, https://doi.org/10.1016/j.ijhydene.2016.01.062.

[15] J. Chan, J.P. Sislian, D. Alexander, Numerically simulated comparative performance of a scramjet and shcramjet at Mach 11, J. Propuls. Power 26 (2010 1125-1134, https://doi.org/10.2514/1.48144.

[16] C.I. Morris, M.R. Kamel, I.G. Stouklov, R.K. Hanson, PLIF imaging of the supersonic reactive flows are projectiles in an expansion tube, in: AIAA 34th Aerosp. Sci. Meet. Exhib., Reno, Nevada, 1996.

[17] M. Kamel, C. Morris, R.K. Hanson, Simultaneous PLIF and schlieren imaging of hypersonic reactive flows around blunted cylinders, in: AIAA 35th Aerosp. Sci. Meet., Reno, Nevada, 1997.

[18] D. Desbordes, L. Hamada, C. Guerraud, Supersonic H2-air combustions behind oblique shock waves, Shock Waves 4 (1995) 339-345, https://doi.org/10.1007/ BF01413876. 
[19] C. Viguier, C. Guerraud, D. Desbordes, H2-air and CH4-air detonations and combustion behind oblique shock waves, Symp. Combust. 25 (1994) 53-59.

[20] H.F. Lehr, Experiments on shock-induced combustion, Astronaut. Acta 17 (1972) 589-597.

[21] L.F. Figueria Da Silva, B. Deshaies, Stabilization of an oblique detonation wave by a wedge: a parametric numerical study, Combust. Flame 121 (2000) 152-166, https://doi.org/10.1016/S0010-2180(99)00141-8.

[22] J. Kasahara, T. Fujiwara, T. Endo, T. Arai, Chapman-Jouguet oblique detonation structure around hypersonic projectiles, AIAA J. 39 (2001) 1553-1561, https:// doi.org/10.2514/2.1480.

[23] S. Bhattrai, H. Tang, Formation of near-Chapman-Jouguet oblique detonation wave over a dual-angle ramp, Aerosp. Sci. Technol. 63 (2017) 1-8, https://doi org/10.1016/j.ast.2016.12.010.

[24] B. Bomjan, S. Bhattrai, H. Tang, Characterization of induction and transition methods of oblique detonation waves over dual-angle wedge, Aerosp. Sci. Technol. 82-83 (2018) 394-401, https://doi.org/10.1016/j.ast.2018.07.038.

[25] M.J. Kaneshige, J.E. Shepherd, Oblique detonation stabilized on a hypervelocity projectile, Symp. Combust. 26 (1996) 3015-3022, https://doi.org/10.1016/ S0082-0784(96)80145-7.

[26] J. Verreault, A.J. Higgins, Initiation of detonation by conical projectiles, Proc. Combust. Inst. 33 (2011) 2311-2318, https://doi.org/10.1016/j.proci.2010.07. 086.

[27] Y. Fang, Z. Zhang, Z. Hu, X. Deng, Initiation of oblique detonation waves induced by a blunt wedge in stoichiometric hydrogen-air mixtures, Aerosp. Sci. Technol. 92 (2019) 676-684, https://doi.org/10.1016/j.ast.2019.06.031.

[28] G. Xiang, X. Li, X. Sun, X. Chen, Investigations on oblique detonations induced by a finite wedge in high altitude, Aerosp. Sci. Technol. 95 (2019) 105451 https://doi.org/10.1016/j.ast.2019.105451.

[29] Y. Liu, X. Han, S. Yao, J. Wang, A numerical investigation of the prompt oblique detonation wave sustained by a finite-length wedge, Shock Waves 26 (2016) 729-739, https://doi.org/10.1007/s00193-016-0626-3.

[30] P. Yang, H.D. Ng, H. Teng, Z. Jiang, Initiation structure of oblique detonation waves behind conical shocks, Phys. Fluids 29 (2017) 086104, https://doi.org $10.1063 / 1.4999482$.

[31] S. Miao, J. Zhou, S. Liu, X. Cai, Formation mechanisms and characteristics of transition patterns in oblique detonations, Acta Astronaut. 142 (2018) 121-129, https://doi.org/10.1016/j.actaastro.2017.10.035.

[32] H.H. Teng, Z.L. Jiang, On the transition pattern of the oblique detonation structure, J. Fluid Mech. 713 (2012) 659-669, https://doi.org/10.1017/jfm.2012.478.

[33] P. Yang, H. Teng, Z. Jiang, H.D. Ng, Effects of inflow Mach number on oblique detonation initiation with a two-step induction-reaction kinetic model, Combust. Flame 193 (2018) 246-256, https://doi.org/10.1016/j.combustflame.2018. 03.026.

[34] H. Teng, Y. Zhang, Z. Jiang, Numerical investigation on the induction zone structure of the oblique detonation waves, Comput. Fluids 95 (2014) 127-131 https://doi.org/10.1016/j.compfluid.2014.03.001.

[35] Y. Liu, Y.-S. Liu, D. Wu, J.-P. Wang, Structure of an oblique detonation wave induced by a wedge, Shock Waves 26 (2016) 161-168, https://doi.org/10.1007/ s00193-015-0600-5.

[36] S. Maeda, J. Kasahara, A. Matsuo, Oblique detonation wave stability around a spherical projectile by a high time resolution optical observation, Combust. Flame 159 (2012) 887-896, https://doi.org/10.1016/j.combustflame.2011. 09.001.

[37] C. Viguier, A. Gourara, D. Desbordes, Three-dimensional structure of stabilization of oblique detonation wave in hypersonic flow, Symp. Combust. 27 (1998) 2207-2214.
[38] K. Iwata, S. Nakaya, M. Tsue, Numerical investigation of the effects of nonuniform premixing on shock-induced combustion, AIAA J. 54 (2016) 1682-1692, https://doi.org/10.2514/1.J054118.

[39] K. Iwata, S. Nakaya, M. Tsue, Wedge-stabilized oblique detonation in an inhomogeneous hydrogen-air mixture, Proc. Combust. Inst. 36 (2016) 2761-2769, https://doi.org/10.1016/j.proci.2016.06.094.

[40] B. Edney, Anomalous Heat Transfer and Pressure Distributions on Blunt Bodies at Hypersonic Speeds in the Presence of an Impinging Shock, Stockholm, Sweden, 1968.

[41] J. Blazek, Computational Fluid Dynamics: Principles and Applications, third ed., Butterworth-Heinemann, Oxford, 2015.

[42] R.W. Houim, B.D. Taylor, Detonation initiation from shock and material interface interactions in hydrogen-air mixtures, Proc. Combust. Inst. 37 (2019) 3513-3520, https://doi.org/10.1016/j.proci.2018.06.037.

[43] H.H. Teng, Z.L. Jiang, H.D. Ng, Numerical study on unstable surfaces of oblique detonations, J. Fluid Mech. 744 (2014) 111-128, https://doi.org/10.1017/jfm. 2014.78.

[44] C. Leung, M.I. Radulescu, G.J. Sharpe, Characteristics analysis of the onedimensional pulsating dynamics of chain-branching detonations, Phys. Fluids 22 (2010) 126101, https://doi.org/10.1063/1.3520188.

[45] G.S. Jiang, C.W. Shu, Efficient implementation of weighted ENO schemes, J. Comput. Phys. 126 (1996) 202-228, https://doi.org/10.1006/jcph.1996.0130.

[46] F. Acker, R.B.R. Borges, B. Costa, An improved WENO-Z scheme, J. Comput. Phys. 313 (2016) 726-753, https://doi.org/10.1016/j.jcp.2016.01.038.

[47] P.L. Roe, Approximate Riemann solvers, parameter vectors, and difference schemes, J. Comput. Phys. 135 (1997) 250-258, https://doi.org/10.1006/jcph. 1997.5705.

[48] B. Einfeld, On Godunov-type methods for gas dynamics, SIAM J. Numer. Anal. 25 (1988) 294-318, https://doi.org/10.1137/0725021.

[49] A.C. Hindmarsh, P.N. Brown, K.E. Grant, S.L. Lee, R. Serban, D.E. Shumaker, C.S Woodward, SUNDIALS: suite of nonlinear and differential/algebraic equation solvers, ACM Trans. Math. Softw. 31 (2005) 363-396, https://doi.org/10.1145/ 1089014.1089020.

[50] C.A. Kennedy, M.H. Carpenter, Additive Runge-Kutta schemes for convectiondiffusion-reaction equations, Appl. Numer. Math. 44 (2003) 139-181, https:// doi.org/10.1016/S0168-9274(02)00138-1.

[51] W. Gropp, E. Lusk, A. Skjellum, Using MPI: Portable Parallel Programming with the Message-Passing Interface, MIT Press, Cambridge, MA, USA, 1994.

[52] A.E. Medvedev, Reflection of an oblique shock wave in a reacting gas with a finite relaxation-zone length, J. Appl. Mech. Tech. Phys. 42 (2001) 211-218, https://doi.org/10.1023/A:1018811516116.

[53] H. Li, A. Chpoun, G. Ben-Dor, Analytical and experimental investigations of the reflection of asymmetric shock waves in steady flows, J. Fluid Mech. 390 (1999) 25-43, https://doi.org/10.1017/S0022112099005169.

[54] D.T. Pratt, J.W. Humphrey, D.E. Glenn, Morphology of standing oblique detonation waves, Jet Propuls. 7 (1991) 837-845, https://doi.org/10.2514/3.23399.

[55] J. Olejniczak, M.J. Wright, G.V. Candler, Numerical study of inviscid shock interactions on double-wedge geometries, J. Fluid Mech. 352 (1997) 1-25, https:// doi.org/10.1017/S0022112097007131.

[56] J. Li, Y. Zhu, X. Luo, On type VI-V transition in hypersonic double-wedge flows with thermo-chemical non-equilibrium effects, Phys. Fluids 26 (2014) 086104, https://doi.org/10.1063/1.4892819.

[57] Z.M. Hu, Y.L. Gao, R.S. Myong, H.S. Dou, B.C. Khoo, Geometric criterion for $\mathrm{RR} \leftrightarrow \mathrm{MR}$ transition in hypersonic double-wedge flows, Phys. Fluids 22 (2010) 016101, https://doi.org/10.1063/1.3276907. 